CLINICAL STUDY

\title{
Ghrelin levels are suppressed and show a blunted response to oral glucose in women with polycystic ovary syndrome
}

\author{
Thomas M Barber, Felipe F Casanueva ${ }^{1}$, Fredrik Karpe, Mary Lage ${ }^{1}$, Stephen Franks ${ }^{2}$, Mark I McCarthy \\ and John A H Wass \\ Oxford Centre for Diabetes, Endocrinology and Metabolism, Churchill Hospital, Old Road, Headington, Oxford OX3 $7 \mathrm{LJ}$, UK, ${ }^{1}$ Endocrine Division, \\ Department of Medicine, Santiago de Compostela University, E-15780 Santiago de Compostela, Spain and ${ }^{2}$ Institute of Reproductive and Developmental \\ Biology, Imperial College (Hammersmith Campus), London W12 ONN, UK \\ (Correspondence should be addressed to T M Barber; Email: tom.barber@drl.ox.ac.uk)
}

\begin{abstract}
Objective: Abnormal ghrelin regulation may influence the development of obesity-associated conditions including polycystic ovary syndrome (PCOS). Our aim was to compare ghrelin regulation between PCOS cases and controls.

Design: We compared serum ghrelin (total) levels, fasting and 30-min post-oral $(75 \mathrm{~g})$ glucose load, between 50 PCOS cases and 28 female controls, including 22 body mass index (BMI)/fat massmatched pairs. All subjects were of UK British/Irish origin.

Methods: Measurements included serum ghrelin (RIA technique (LINCO Research, St Charles MO, USA)), fat mass, serum testosterone, fasting serum insulin and plasma glucose levels. Insulin sensitivity was calculated as the homeostasis model assessment of insulin resistance (HOMA2 IR). Results: Fasting serum ghrelin levels were significantly lower in PCOS cases versus BMI/fat massmatched controls (geometric mean (s.D. range), 1104 pg/ml (764-1595) vs 1756 pg/ml (1314-2347) respectively; $\left.P=2.3 \times 10^{-4}\right)$. Ghrelin suppression following oral glucose load was significantly blunted in PCOS cases versus BMI/fat mass-matched controls (geometric mean ghrelin suppression (s.D. range), $160 \mathrm{pg} / \mathrm{ml}(88-289)$ vs $424 \mathrm{pg} / \mathrm{ml}(220-818)$ respectively; $\left.P=2.0 \times 10^{-4}\right)$. Whole-group comparisons ( 50 PCOS cases versus 28 controls) adjusted for fat mass and age revealed similar results. In PCOS cases, there was a significant negative correlation between fasting serum ghrelin and HOMA2 IR $\left(r^{2}=-0.40, P=5.7 \times 10^{-3}\right)$. Following adjustment for HOMA2 IR, fat mass and age, comparisons between the whole groups of PCOS cases and controls revealed attenuated but significant differences in fasting serum ghrelin $\left(P=1.3 \times 10^{-3}\right)$ and ghrelin suppression $\left(P=1.8 \times 10^{-3}\right)$.

Conclusions: In women with PCOS, serum ghrelin levels are suppressed, showing a negative relationship with HOMA2 IR and a blunted response to oral glucose.

European Journal of Endocrinology 158 511-516
\end{abstract}

\section{Introduction}

Polycystic ovary syndrome (PCOS) characteristically presents with oligoamenorrhoea and hyperandrogenic features (1). Obesity is an important factor implicated in the aetiology of PCOS (2). Furthermore, the frequent concurrence of obesity and PCOS places this group of women at particularly high risk of developing an adverse metabolic profile, including insulin resistance (3). Factors that may play a role in the development of obesity, or in its translation to PCOS, are therefore relevant to PCOS pathogenesis.

Ghrelin is a novel 28-amino acid peptide hormone that is secreted by endocrine cells in the stomach (4). Ghrelin is a natural ligand of the growth hormone secretagogue receptor, and has been demonstrated to enhance appetite, reduce fat utilisation and promote adiposity following central or peripheral administration to both rodents and humans $(5,6)$. Serum concentrations of ghrelin typically increase on fasting and decrease following food intake $(6,7)$. It is therefore imperative to obtain measurements of serum ghrelin levels in both fasting and non-fasting states for a full assessment of ghrelin regulation. The potent orexigenic effect of ghrelin supports the hypothesis that ghrelin may act as a trigger for the initiation of meals (8), and the postprandial suppression of ghrelin may impart a satiating effect that is important for meal termination (7).

There have been few studies of ghrelin in PCOS, and most have focused on fasting (total) ghrelin levels (9-13). These have yielded inconsistent and conflicting data to support both normal (14) and abnormal (significantly lower) fasting serum ghrelin levels in women with PCOS versus controls (9-11). In a couple of studies, a significant negative correlation between fasting serum ghrelin and homeostasis model assessment of insulin 
resistance (HOMA2 IR; a measure of insulin sensitivity) has been demonstrated $(9,11)$. However, only one previous study has assessed postprandial serum ghrelin levels in PCOS (15), and the lack of equivalent postprandial data in other studies represents a significant limitation of the current literature. Furthermore, several of the previous studies have involved relatively low numbers of subjects $(11,15)$.

The aim of our study was to perform a detailed examination of fasting and post-oral glucose load ghrelin dynamics in women with and without PCOS, relating these findings to the characteristics of the groups (including differences in body mass index (BMI)/fat mass and measures of insulin sensitivity). Given the role of ghrelin in appetite regulation and promotion of adiposity (8), and the association between obesity and PCOS (2), we tested the hypothesis that women with PCOS have abnormal serum levels of ghrelin. Given that PCOS is associated with insulin resistance (2) and that ghrelin has been shown to be related to measures of insulin sensitivity in some studies $(9,11)$, a further aim was to explore this relationship.

\section{Subjects and methods}

\section{Subjects}

All cases and controls were white, of British/Irish origin, pre-menopausal and post-menarchal women. Exclusion factors included pregnancy and diabetes. All cases $(n=50)$ had a confirmed diagnosis of PCOS based on Rotterdam diagnostic criteria (1), including magnetic resonance imaging (MRI)-confirmed polycystic ovarian (PCO) morphology $(1,16)$. The majority of the PCOS cases $(n=44)$ also fulfilled both of the other two Rotterdam diagnostic criteria: oligoamenorrhoea (inter-menstrual interval greater than 42 days) and hyperandrogenism (defined clinically as a FerrimanGallwey score of $\geq 8$ and/or the presence of androgenic alopecia, and biochemically as a serum testosterone concentration $\geq 2.36 \mathrm{nmol} / \mathrm{l}$ and/or free androgen index $\geq 8.98$ ) (1). In addition to the presence of PCO morphology, the remaining six PCOS cases each fulfilled one of the other two Rotterdam diagnostic criteria: five had hyperandrogenism but regular menses, and one had oligoamenorrhoea but normoandrogenaemia. The cut-off values for defining hyperandrogenaemia were taken from the distribution $($ mean +2 s.D. $)$ for testosterone and SHBG measured with the same assays in the control women. Other causes of hyperandrogenaemia had been excluded previously (1). PCOS cases were recruited from general endocrine and PCOS clinics at the Churchill and John Radcliffe Hospitals, Oxford.

The control group of women $(n=28)$ were recruited from Oxford Biobank. All the control women had regular menstrual cycles and MRI-confirmed normal ovarian morphology, and none had clinical or biochemical evidence of hyperandrogenism (1). Therefore, none of the control women fulfilled any of the Rotterdam diagnostic criteria for PCOS, and a diagnosis of PCOS could be excluded (1). The control group was selected from a larger group of control women $(n=40)$, all of whom had regular menstrual cycles and normoandrogenaemia. However, 12 of the women in the putative control group were excluded from the study on the basis of MRI-proved PCO morphology. A minority of the PCOS cases $(n=21)$ had been taking metformin, and this was stopped for 1 week prior to the blood tests. No other confounding medications (including hormonal preparations) had been taken by any of the subjects within 3 months of the study.

It was not possible to obtain a perfect match for BMI and age between the whole groups of PCOS cases and controls (data shown in Table 1). To limit a potential confounding effect of disparate BMI/fat mass between groups of PCOS cases and controls, we adjusted for these differences in the whole-group comparisons (50 PCOS cases versus 28 controls), but also defined a subset of BMI/fat mass-matched pairs of PCOS cases and controls, selected from their respective whole groups. Within the $22 \mathrm{BMI} /$ fat mass-matched pairs, we also sought to reduce the metabolic heterogeneity associated with inclusion of multiple PCOS phenotypic subgroups (3) by ensuring that all 22 PCOS cases fulfilled all three Rotterdam-proscribed PCOS diagnostic criteria (PCO morphology, oligoamenorrhoea and hyperandrogenism) (1). All clinical investigations were conducted in accordance with the guidelines in the Helsinki Declaration of 1975, as revised in 2000, and the study was approved by the relevant ethics committee in the United Kingdom. All subjects provided fully informed consent.

\section{Anthropometric and biochemical evaluation}

A body composition analyser (TBF-305) was used to measure fat mass. Tanita bioelectrical impedance analysis technology (an established well-validated technique for measurement of fat mass (17)) was used for this purpose (Tanita UK Ltd, Middlesex, UK). BMI and bio-impedance-measured fat mass were highly correlated in our study $\left(r^{2}=0.95, P=1.1 \times 10^{-52}\right)$. Serum ghrelin (total) levels were measured using an RIA technique (LINCO Research, St Charles MO, USA). The intra-assay coefficient of variation (CV) for the ghrelin assay was 5.6-8\%. Serum (specific) insulin was analysed using an automated immunometric assay, plasma glucose with a hexokinase assay (ADVIA 2400 analyser, intra-assay $\mathrm{CV}<1 \%$ ) and non-esterified fatty acids (NEFA) with an enzymatic colorimetric method (NEFA C kit; Wako Chemicals, Alpha Laboratories, Eastleigh, Hampshire, UK). Insulin sensitivity was assessed using the HOMA2 IR (Oxford Diabetes Trials Unit calculator, www.dtu.ox.ac.uk), which has previously been shown to correlate well with measures of insulin sensitivity derived from the 


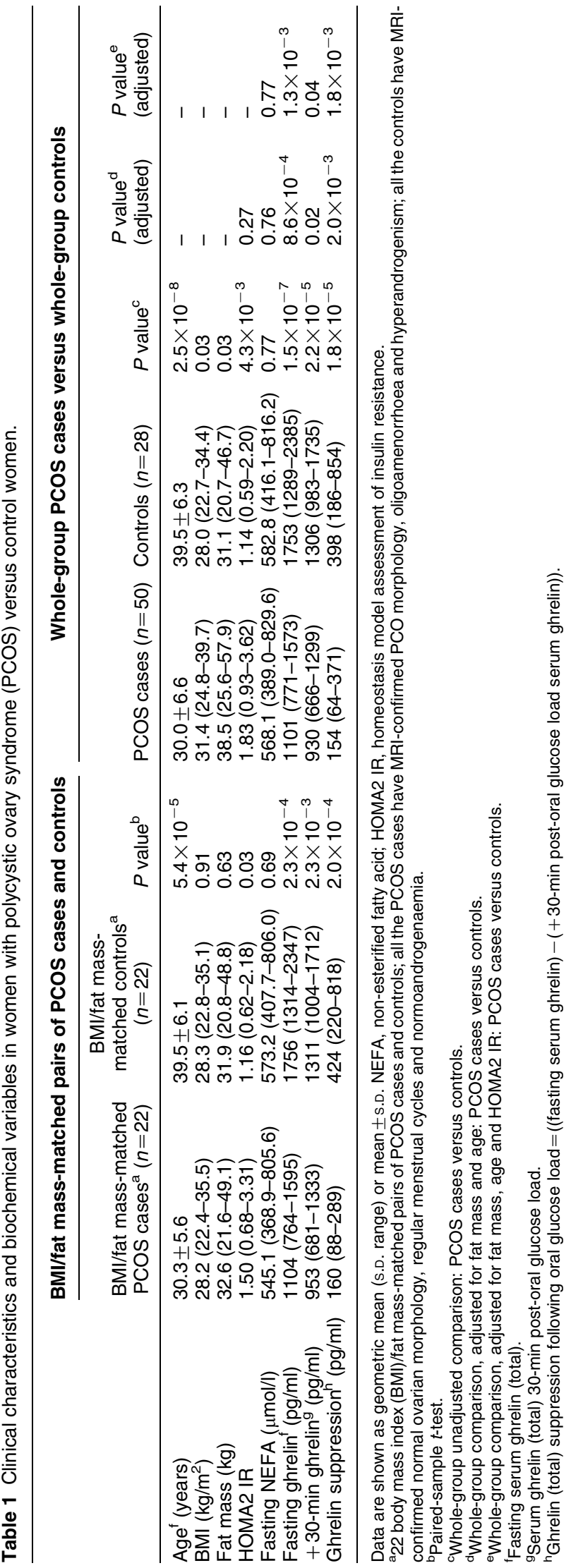

euglycaemic-hyperinsulinaemic clamp (18). As a measure of acute insulin secretion during the standard $(75 \mathrm{~g})$ oral glucose tolerance test, the insulinogenic index (InsI) was calculated using the equation: $\mathrm{InsI}=$ $((+30$-min insulin-fasting insulin $) /(+30$-min glucosefasting glucose)) (16). As a further measure of insulin secretion, the insulin disposition index (DI) was calculated using the equation: $\mathrm{DI}=(\mathrm{InsI} \times \mathrm{HOMA} 2$ IR) (16). Serum testosterone was analysed using a direct (competitive) chemiluminescent assay (ADVIA centaur analyser, intra-assay CV $<7 \%$ ) and SHBG using an immunometric assay (Immulite 2000 analyser, intra-assay $\mathrm{CV}<4 \%$ ). All blood samples were taken at $0900 \mathrm{~h}$ following an overnight fast. To limit the potential for serum ghrelin levels to vary during the menstrual cycle (19), all the controls and the five PCOS cases with regular menstrual cycles had blood taken during the follicular phase (days 2-5) of the menstrual cycle. This was not possible, however, for the majority of the PCOS cases who had irregular or absent menses.

\section{Statistical analyses}

For comparisons of the pair-matched groups of PCOS cases and controls, we used paired-sample $t$-tests. For comparisons of the whole groups, we used independentsample $t$-tests (unadjusted, adjusted for fat mass and age, and adjusted for fat mass, age and HOMA2 IR). To explore further the relationships of fasting serum ghrelin with other biochemical indices in each of the whole groups, bivariate Pearson's correlation analyses were performed. All analyses were conducted in SPSS (version 12.0 for Windows; SPSS Inc., Chicago, IL, USA). All variables were skewed. Therefore, prior to statistical analyses, all variables underwent logarithmic transformation, following which normality of their distributions was achieved. $P<0.05$ was considered significant. We had $>80 \%$ power to detect a betweengroup difference exceeding $86 \%$ and $67 \%$ of an s.D. for serum ghrelin levels in the BMI/fat mass-matched PCOS case-control pairs and whole-group comparisons respectively $(\alpha=0.05)$.

\section{Results}

Data are shown in Table 1. Paired-sample comparisons revealed that serum ghrelin (total) levels (both fasting and +30-min post-oral glucose load) were significantly lower in women with PCOS compared with BMI/fat massmatched control women (fasting serum ghrelin geometric mean (s.D. range), $1104 \mathrm{pg} / \mathrm{ml}$ (764-1595) vs $1756 \mathrm{pg} /$ $\mathrm{ml}(1314-2347)$ respectively, $P=2.3 \times 10^{-4} ;+30-\mathrm{min}$ serum ghrelin $953 \mathrm{pg} / \mathrm{ml}(681-1333)$ vs $1311 \mathrm{pg} / \mathrm{ml}$ (1004-1712) respectively, $P=2.3 \times 10^{-3}$; Table 1). Paired-sample comparisons also revealed significantly 
lower post-oral glucose load ghrelin suppression ((fasting serum ghrelin $)-(+30$-min post-oral glucose load serum ghrelin)) in PCOS cases versus BMI/fat mass-matched control women (geometric mean ghrelin suppression (s.D. range), $160 \mathrm{pg} / \mathrm{ml}(88-289)$ vs $424 \mathrm{pg} / \mathrm{ml}$ (220-818) respectively, $P=2.0 \times 10^{-4}$; Table 1$)$.

Analyses of the full-sample set (50 PCOS cases versus 28 controls) generated similar results (Table 1). Differences in the whole-group analyses for PCOS cases and controls remained significant following adjustment for fat mass and age, including fasting serum ghrelin (adjusted $P=8.6 \times 10^{-4}$ ), +30 -min serum ghrelin (adjusted $P=0.02$ ) and ghrelin suppression (adjusted $P=2.0 \times 10^{-3}$; Table 1 ). Similar results were obtained when using relative rather than absolute changes in serum ghrelin (data not shown). Differences in the whole-group analyses remained significant following adjustment for fat mass, age and HOMA2 IR, including fasting serum ghrelin (adjusted $P=1.3 \times$ $\left.10^{-3}\right),+30$-min serum ghrelin (adjusted $P=0.04$ ) and ghrelin suppression (adjusted $P=1.8 \times 10^{-3}$; Table 1 ).

Since there had been no significant relationships between serum ghrelin (fasting, + 30-min and ghrelin suppression) and age in either PCOS cases or controls, differences in age between the groups are unlikely to explain the differences in ghrelin regulation. Prior use of metformin was also unlikely to be confounding as comparison of the subgroup of PCOS cases naïve to prior use of metformin $(n=29)$ versus the control group made no difference to the results.

Given the significant differences in ghrelin regulation between PCOS cases and controls, we also explored the relationships between fasting serum ghrelin and other metabolic indices in each of the groups. In the PCOS cases group $(n=50)$, there were significant negative correlations between the fasting serum ghrelin and the following indices: HOMA2 IR $\left(r^{2}=-0.40, P=5.7 \times\right.$ $10^{-3}$, shown graphically in Fig. 1), fasting serum insulin $\left(r^{2}=-0.40, P=6.1 \times 10^{-3}\right)$, DI $\left(r^{2}=-0.44\right.$, $\left.P=2.3 \times 10^{-3}\right)$ and InsI $\left(r^{2}=-0.31, P=0.04\right)$. In the control group, equivalent correlations with fasting serum ghrelin were weaker and failed to reach statistical significance: HOMA2 IR $\left(r^{2}=-0.16, P=0.41\right)$, fasting serum insulin $\left(r^{2}=-0.07, P=0.66\right)$, DI $\left(r^{2}=-0.14, \quad P=0.49\right)$ and InsI $\left(r^{2}=-0.06\right.$, $P=0.78)$. There were no correlations between fasting serum ghrelin and plasma NEFA levels in PCOS cases $\left(r^{2}=-0.08, P=0.58\right)$ or controls $\left(r^{2}=0.08, P=0.60\right)$, nor between fasting serum ghrelin and serum testosterone levels (PCOS cases: $r^{2}=-0.13, P=0.36$; controls: $\left.r^{2}=-0.23, P=0.24\right)$.

\section{Discussion}

We provide clear evidence that ghrelin regulation is abnormal in a group of well-phenotyped women with

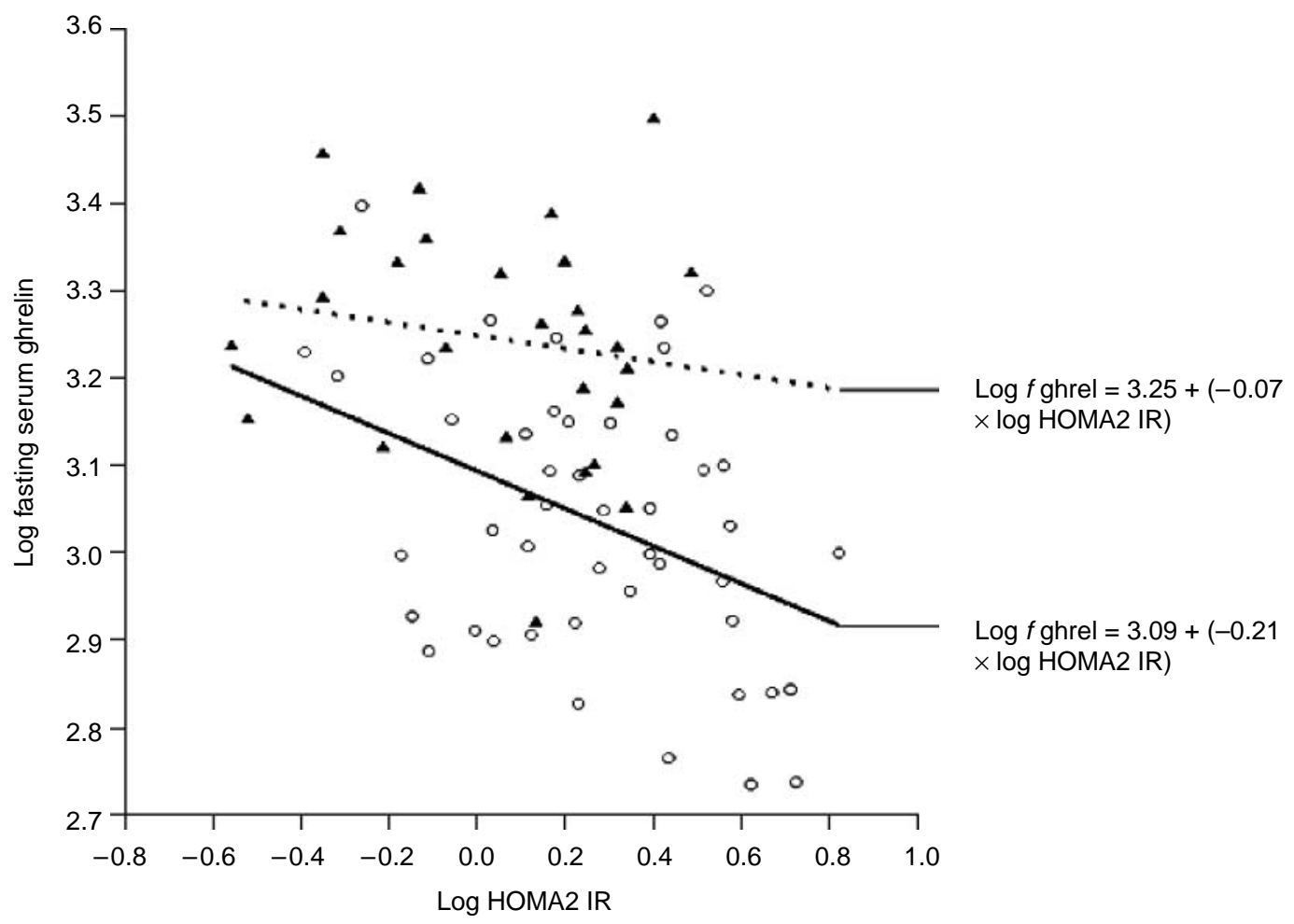

Figure 1 Scatterplot showing correlation of fasting serum ghrelin with HOMA2 IR. (Women with PCOS are represented with open circles (regression line unbroken) and control women with filled triangles (regression line dashed)). 
PCOS. The differences in serum ghrelin levels (both fasting and +30-min post-oral glucose load) between cases and controls were significant for the whole-group comparisons (both unadjusted and adjusted for fat mass, age and HOMA2 IR) and in a sub-analysis in which PCOS cases and controls were matched for $\mathrm{BMI} /$ fat mass. We speculate that potential implications of these data in women with PCOS include a role for ghrelin dysregulation in the development of and/or response to obesity.

In normal physiology, serum ghrelin levels vary markedly before and after a meal (7). To fully appreciate the dynamics (and possible abnormalities) of ghrelin regulation, it is important to measure serum ghrelin levels in both fasting and non-fasting states. There have been very few studies that have measured non-fasting ghrelin levels in PCOS. Our findings of blunted ghrelin suppression following an oral glucose load in PCOS are consistent with a previous (smaller) study that used high/standard protein meals instead of oral glucose (15).

Intravenous infusion of ghrelin in healthy humans has been shown to enhance appetite and increase energy consumption from a free-choice buffet (8). Chronic ghrelin administration has also been demonstrated to result in weight gain and adiposity in rodents (17). Although there is a frequent occurrence of obesity in women with PCOS, it is not clear whether and how suppressed fasting serum ghrelin levels impact on preprandial appetite regulation and energy balance in PCOS, and what, if any effect this would have on fat mass. It is possible that ghrelin dysregulation contributes towards the development of obesity in women with PCOS, through a mechanism whereby blunted postprandial ghrelin suppression leads to a reduction in the postprandial satiating effect of acute food intake, and a tendency towards over-eating during meals. In support of this hypothesis is the demonstration in a previous study of a reduction of postprandial satiety in women with PCOS, who also had blunted postprandial ghrelin suppression (15).

It is also possible that suppressed serum ghrelin levels in women with PCOS represent a physiological response to obesity, designed to minimise appetite and caloric intake. It has been demonstrated previously that obesity per se (20) and insulin resistance (21) are both associated with suppressed serum ghrelin levels. However, our data do not support the notion that ghrelin suppression in PCOS is purely due to the frequent concurrence of obesity (and insulin resistance) in women with PCOS, but rather that PCOS-associated factors independent of obesity and insulin resistance are important. Alternative explanations for abnormal ghrelin regulation in women with PCOS include a direct effect of ghrelin on the development of PCOS independent of any possible effect on appetite or fat mass, or that ghrelin dysregulation represents a manifestation of $\mathrm{PCOS}$ rather than having a role in its pathogenesis.
Although causality cannot be inferred, the significant negative association between fasting serum ghrelin and HOMA2 IR, and between fasting serum ghrelin and measures of acute insulin secretion following an oral glucose load (InsI and DI), suggests that the inherent insulin resistance that is a feature of PCOS plays a role in ghrelin suppression in these women. However, our data (adjusted for fat mass, age and HOMA2 IR) also suggest that mechanism(s) independent of insulin resistance, at least in part, influences ghrelin dysregulation in women with PCOS. It has been proposed that fasting NEFA levels could mediate the inter-relationship between fasting serum ghrelin and insulin sensitivity in PCOS (9). However, our data do not support this hypothesis, given the lack of significant differences in fasting plasma NEFA levels between PCOS cases and controls, and the lack of association between fasting serum ghrelin and plasma NEFA levels in the PCOS cases group. Our data also do not support the notion that serum testosterone is implicated in ghrelin regulation in women with PCOS, given the lack of association between fasting serum ghrelin and serum testosterone levels in the PCOS cases group.

To summarise, we provide the strongest evidence yet presented that ghrelin dysregulation, manifesting as suppression of fasting serum ghrelin levels and blunting of the early response of serum ghrelin to an oral glucose load, is a feature of women with PCOS. Our data show that in PCOS, there is a negative association between fasting serum ghrelin and HOMA2 IR. Our data also suggest an intrinsic abnormality of ghrelin regulation in PCOS not attributable to obesity or insulin resistance alone, although this latter hypothesis requires further clarification with more dynamic measures of insulin sensitivity. The mechanism(s) linking abnormal ghrelin regulation with PCOS should be a focus for future research.

\section{Acknowledgements}

We acknowledge the patients and nurses who contributed to the ascertainment of the various clinical samples used in this study. Clinical Research Fellowship was awarded by Novo Nordisk (to T M B).

\section{References}

1 The Rotterdam ESHRE/ASRM-sponsored PCOS consensus workshop group. Revised 2003 consensus on diagnostic criteria and long-term health risks related to polycystic ovary syndrome (PCOS). Human Reproduction 200419 41-47.

2 Barber TM, McCarthy MI, Wass JA \& Franks S. Obesity and polycystic ovary syndrome. Clinical Endocrinology $2006 \mathbf{6 5}$ 137-145.

3 Barber TM, Wass JA, McCarthy MI \& Franks S. Metabolic characteristics of women with polycystic ovaries and oligoamenorrhoea but normal androgen levels: implications for the management of polycystic ovary syndrome. Clinical Endocrinology $200766513-517$. 
4 Kojima M, Hosoda H, Date Y, Nakazato M, Matsuo H \& Kangawa K. Ghrelin is a growth-hormone-releasing acylated peptide from stomach. Nature $1999 \mathbf{4 0 2} 656-660$.

5 Tschop M, Smiley DL \& Heiman ML. Ghrelin induces adiposity in rodents. Nature $2000 \mathbf{4 0 7} 908-913$.

6 Cummings DE. Ghrelin and the short- and long-term regulation of appetite and body weight. Physiology and Behavior 200689 71-84.

7 Tschop M, Wawarta R, Riepl RL, Friedrich S, Bidlingmaier M, Landgraf R \& Folwaczny C. Post-prandial decrease of circulating human ghrelin levels. Journal of Endocrinological Investigation 2001 24 RC19-RC21.

8 Wren AM, Seal LJ, Cohen MA, Brynes AE, Frost GS, Murphy KG, Dhillo WS, Ghatei MA \& Bloom SR. Ghrelin enhances appetite and increases food intake in humans. Journal of Clinical Endocrinology and Metabolism 2001865992.

9 Schofl C, Horn R, Schill T, Schlosser HW, Muller MJ \& Brabant G. Circulating ghrelin levels in patients with polycystic ovary syndrome. Journal of Clinical Endocrinology and Metabolism 2002 87 4607-4610.

10 Panidis D, Farmakiotis D, Koliakos G, Rousso D, Kourtis A, Katsikis I, Asteriadis C, Karayannis V \& Diamanti-Kandarakis E. Comparative study of plasma ghrelin levels in women with polycystic ovary syndrome, in hyperandrogenic women and in normal controls. Human Reproduction $2005202127-2132$.

11 Pagotto U, Gambineri A, Vicennati V, Heiman ML, Tschop M \& Pasquali R. Plasma ghrelin, obesity, and the polycystic ovary syndrome: correlation with insulin resistance and androgen levels. Journal of Clinical Endocrinology and Metabolism $2002 \mathbf{8 7}$ 5625-5629.

12 Wasko R, Komarowska H, Warenik-Szymankiewicz A \& Sowinski J. Elevated ghrelin plasma levels in patients with polycystic ovary syndrome. Hormone and Metabolic Research 200436 170-173.

13 Gambineri A, Pagotto U, Tschop M, Vicennati V, Manicardi E, Carcello A, Cacciari M, De Iasio R \& Pasquali R. Anti-androgen treatment increases circulating ghrelin levels in obese women with polycystic ovary syndrome. Journal of Endocrinological Investigation 200326 629-634.
14 Orio F Jr, Lucidi P, Palomba S, Tauchmanova L, Cascella T, Russo T, Zullo F, Colao A, Lombardi G \& De Feo P. Circulating ghrelin concentrations in the polycystic ovary syndrome. Journal of Clinical Endocrinology and Metabolism $2003 \mathbf{8 8} 942-945$.

15 Moran LJ, Noakes M, Clifton PM, Wittert GA, Tomlinson L, Galletly C, Luscombe ND \& Norman RJ. Ghrelin and measures of satiety are altered in polycystic ovary syndrome but not differentially affected by diet composition. Journal of Clinical Endocrinology and Metabolism $2004893337-3344$.

16 Adams J, Polson DW \& Franks S. Prevalence of polycystic ovaries in women with anovulation and idiopathic hirsutism. British Medical Journal (Clinical Research Ed.) 1986293 355-359.

17 Ritchie JD, Miller CK \& Smiciklas-Wright H. Tanita foot-to-foot bioelectrical impedance analysis system validated in older adults. Journal of the American Dietetic Association $2005 \mathbf{1 0 5}$ 1617-1619.

18 Mari A, Pacini G, Brazzale AR \& Ahren B. Comparative evaluation of simple insulin sensitivity methods based on the oral glucose tolerance test. Diabetologia $2005 \mathbf{4 8} 748-751$.

19 Barkan AL, Dimaraki EV, Jessup SK, Symons KV, Ermolenko M \& Jaffe CA. Ghrelin secretion in humans is sexually dimorphic, suppressed by somatostatin, and not affected by the ambient growth hormone levels. Journal of Clinical Endocrinology and Metabolism $2003 \mathbf{8 8} 2180-2184$.

20 Shiiya T, Nakazato M, Mizuta M, Date Y, Mondal MS, Tanaka M, Nozoe S, Hosoda H, Kangawa K \& Matsukura S. Plasma ghrelin levels in lean and obese humans and the effect of glucose on ghrelin secretion. Journal of Clinical Endocrinology and Metabolism 200287 240-244.

21 Katsuki A, Urakawa H, Gabazza EC, Murashima S, Nakatani K, Togashi K, Yano Y, Adachi Y \& Sumida Y. Circulating levels of active ghrelin is associated with abdominal adiposity, hyperinsulinemia and insulin resistance in patients with type 2 diabetes mellitus. European Journal of Endocrinology 2004151 573-577.

Received 12 December 2007

Accepted 27 December 2007 